\title{
Subthreshold Erosion of an Organic Polymer Induced by Multiple Shots of an X-Ray Free-Electron Laser
}

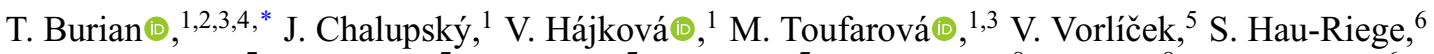 \\ J. Krzywinski, ${ }^{7}$ J.D. Bozek, ${ }^{7}$ C. Bostedt, ${ }^{7}$ A.T. Graf, ${ }^{7}$ U.F. Jastrow, ${ }^{8}$ S. Kreis, ${ }^{8}$ R.A. London, ${ }^{6}$ \\ M. Messerschmidt, ${ }^{9}$ S. Moeller $\odot,{ }^{7}$ R. Sobierajski $\odot,{ }^{10} \mathrm{~K}$. Tiedtke, ${ }^{8}$ M. de Grazia, ${ }^{11}$ T. Auguste, ${ }^{12}$ \\ B. Carré, ${ }^{12}$ S. Guizard, ${ }^{12}$ H. Merdji®,$^{12}$ N. Medvedev, ${ }^{1,3}$ and L. Juha ${ }^{1,3}$ \\ ${ }^{1}$ Department of Radiation and Chemical Physics, Institute of Physics, Czech Academy of Sciences, Na Slovance 2, \\ 18221 Prague 8, Czech Republic \\ ${ }^{2}$ Department of Surface and Plasma Physics, Faculty of Mathematics and Physics, Charles University in Prague, \\ V Holešovičkách 2, 18000 Prague 8, Czech Republic \\ ${ }^{3}$ Laser Plasma Department, Institute of Plasma Physics, Czech Academy of Sciences, Za Slovankou 3, \\ 18200 Prague 8, Czech Republic \\ ${ }^{4}$ Department of Spectroscopy, J. Heyrovsky Institute of Physical Chemistry, Czech Academy of Sciences, \\ Dolejškova 3, 18223 Prague 8, Czech Republic \\ ${ }^{5}$ Department of Dielectrics, Institute of Physics, Czech Academy of Sciences, Na Slovance 2, 18221 Prague 8 , \\ Czech Republic \\ ${ }^{6}$ Lawrence Livermore National Laboratory, 7000 East Avenue, Livermore, California 94550, USA \\ ${ }^{7}$ SLAC National Accelerator Laboratory, 2575 Sand Hill Road, Menlo Park, California 94025, USA \\ ${ }^{8}$ Deutsches Elektronen-Synchrotron DESY, Notkestrasse 85, D-22603 Hamburg, Germany \\ ${ }^{9}$ BioXFEL STC, 700 Ellicott Street, Buffalo, New York 14203, USA \\ ${ }^{10}$ Institute of Physics Polish Academy of Sciences, Al. Lotników 32/46, PL-02-668 Warsaw, Poland \\ ${ }^{11}$ Service Couches Minces, Saint-Gobain Recherche, 93303 Aubervilliers, France \\ ${ }^{12}$ Laboratoire Interactions, Dynamiques et Lasers (LIDYL), CEA, CNRS, Université Paris-Saclay, \\ CEA Saclay 91191 Gif-sur-Yvette France
}

(Received 13 August 2019; revised 20 March 2020; accepted 25 June 2020; published 22 September 2020)

Solids irradiated by energetic photons can be eroded in two modes, depending on the radiation intensity. High average, low-peak power sources, e.g., synchrotron radiation and high-order harmonics, induce desorption of the material at a low etch rate. In contrast, high-peak-power radiation from extreme ultraviolet and x-ray lasers usually causes a massive removal of the material even by a single shot. In this contribution, an effective material erosion is reported in PMMA exposed to multiple accumulated pulses generated by the free-electron x-ray-laser Linac Coherent Light Source (LCLS, tuned at a photon energy of $830 \mathrm{eV}$ in this study, operated in Menlo Park at Stanford, CA, USA) at a fluence below the single-pulse ablation threshold. The effect is caused by polymer-chain scissions initiated by single photons carrying enough energy to break the $\mathrm{C}-\mathrm{C}$ bounds. High efficiency of the erosion is supposed to occur due to a correlation of the single-photon effects. The subthreshold damage exhibits a nonlinear dose dependence resulting from a competition between chain scissions and cross-linking processes. The cross-linking is proven by Raman spectroscopy of the irradiated polymer. Two theoretical models of the x-ray free-electron-laser-induced erosion are suggested, which provide an excellent agreement with the experimental results.

DOI: 10.1103/PhysRevApplied.14.034057

\section{INTRODUCTION}

For several decades, it has been known that extreme ultraviolet (XUV) and x-ray photons, usually delivered by a synchrotron radiation source, carry enough energy to decompose irradiated polymers into tiny volatile pieces through a single-photon absorption, i.e., at relatively

\footnotetext{
*burian@fzu.cz
}

low peak power [1]. This phenomenon is frequently called direct photoetching (less often vacuum etching, photon-induced desorption, etc.). When conventional, long-wavelength radiation interacts with matter, one refers to the effect as laser desorption [2].

Recent advent of the XUV and x-ray laser technology [3-9] makes it possible to investigate the interaction of short-wavelength radiation at much higher intensities than was available with earlier sources. Above a certain fluence, 
a single-shot ablation takes place - a collective action of all photons in the pulse leads to the removal of a massive layer of irradiated material [10].

Energetic XUV and x-ray photons result in a specific character of both the desorption and the ablation [10,11]. Experiments at large facilities especially with shortwavelength self-amplified spontaneous emission (SASE) free-electron lasers (FELs), covering a very wide range of fluences and intensities, led to distinguishing more than the two above-mentioned modes of radiation damage. Irradiation at FLASH, a soft x-ray radiation source, revealed damage patterns related to desorption, intermediate, and ablation processes even in a single shot [11], which is not possible using an ensemble of low-energy photons provided by a conventional long-wavelength laser.

Only a limited number of experiments were devoted to the study of XUV and x-ray-laser-induced desorption where the sample was exposed to multiple pulses at a fluence below the single-shot ablation threshold. Both inorganic [12-16] and organic [17] materials were exposed to XUV and $\mathrm{x}$-ray laser radiation emitted from either plasma [16] or e-beam- $[12-15,17]$ based sources under such irradiation conditions. However, no paper has so far been published connecting an etch rate with radiation-chemical processes occurring in the sample. This is the aim of this study.

\section{EXPERIMENT}

Linac Coherent Light Source (LCLS operated at SLAC, Stanford, CA, USA) [18] is used as a source of intense $\mathrm{x}$-ray radiation in this study. LCLS is a free-electron $\mathrm{x}$ ray laser [4-6,19] tunable from 1.5 to $0.15 \mathrm{~nm}(825 \mathrm{eV}-$ $8250 \mathrm{eV})$ [20]. Irradiation of PMMA (5- $\mu$ m-thick layer of PMMA spin coated on $10 \times 20 \mathrm{~mm}^{2}$ Si slab; Silson, UK) is carried out with $830-\mathrm{eV}$ photons. The pulse duration fluctuated around $100 \mathrm{fs}$ FWHM.

Energy content of the $3 \omega$ component of the laser spectrum is estimated to be below $1 \%$ of the total pulse energy. The influence of this parasitic radiation on the process of material removal is further lowered by the substantially longer absorption length in PMMA $(39.71 \mu \mathrm{m})$ compared to the one for the fundamental $(1.85 \mu \mathrm{m})$ frequency [21]. Hence, the presence of the $3 \omega$ radiation in the spectrum is assumed not to affect the process of ablation but it might play a significant role in the subthreshold effects due to the higher photon energy $(2.505 \mathrm{keV})$ of the third-harmonic radiation.

The LCLS beam is focused on the PMMA surface by Kirkpatrick-Baez mirrors with a focal length of $1.2 \mathrm{~m}$. The focusing system guarantees the beam diameter at the sample surface less than $3 \mu \mathrm{m}$ in the tight focus [22]. The samples are mounted on an $x y z$ positioning stage in the atomic, molecular, and optical physics (AMO) experimental station [23]. Since they are kept in the evacuated interaction chamber for a prolonged period of time, the PMMA near-surface layer can be considered as free of molecular oxygen. Pulse energy is measured by photoionization gas monitor detector (GMD). The average pulse energy in an unattenuated beam is about $1 \mathrm{~mJ}$. The pulse energy is varied by gas attenuators to adjust fluence levels above and below the single-shot ablation threshold. A repetition rate in our experiment is typically $30 \mathrm{~Hz}$ (the maximum repetition rate available at the facility is $120 \mathrm{~Hz}$ [23]). All the samples are irradiated under normal incidence conditions.

Irradiated PMMA surfaces are investigated using a Nomarski optical microscope (BX51M DIC microscope, Olympus, Japan) and by an AFM microscope in tapping mode (D3100 NanoScope Dimension controlled by NanoScope IV Control Station, Veeco, USA). To identify possible structural changes induced in the PMMA layer by LCLS radiation, Raman measurements are performed in the usual backscattering geometry, using a Raman spectrometer (Renishaw Ramascope, UK) equipped with a CCD camera and a Leica microscope DMLP. Typically, $\mathrm{Ar}^{+}$-laser $(514.5 \mathrm{~nm})$ spots with a diameter of $4 \mu \mathrm{m}$ are used. This tool enables us to probe selected locations on the sample surface.

\section{MODELS}

Experimental results are compared with the etch-rate values calculated by means of two simple rate models of the erosion process, which consider a sequence of $n$ laser pulses of time duration $\tau$ (s) separated in time by $T$ (s). As an extension, we introduce separated terms for $1 \omega$ and $3 \omega$ components in order to handle the simultaneous action of these spectral parts.

\section{A. Model A}

The first model (below referred to as model A) describes the evolution of a crater along the beam path in the PMMA sample in two phases. First, within the pulse duration the radiation is absorbed by the PMMA in accordance with the Lambert-Beer law. Intact molecules decompose due to the chain-scission process with the cross section $\xi\left(\mathrm{cm}^{2}\right)$, the density of intact molecules (containing only single bonds in the main chain) $n_{\mathrm{SB}}\left(\mathrm{cm}^{-3}\right)$ decreases while the end links and volatile fragments are produced with densities $n_{B}$ $\left(\mathrm{cm}^{-3}\right)$ and $n_{\text {frag }}\left(\mathrm{cm}^{-3}\right)$, respectively, where the total density of fragments is then given as a sum of the end links and double bonds (each formed double bond liberates two fragments):

$$
n_{\text {frag }}=n_{B}+2 n_{\mathrm{DB}}
$$

The density of intact molecules can then be written in a form of a conservation law:

$$
n_{\mathrm{SB}}=n_{0}-a n_{B}-2 a n_{\mathrm{DB}}=n_{0}-a n_{\mathrm{frag}},
$$


where $a$ is a parameter showing how much the intact molecule is degraded with each chain-scission event. Assuming only one of four single bonds per molecule is broken in a single event, $a$ is equal to 0.25 .

The proposed model is static, i.e., all the molecule transformations are local, the fragments stay close to the point of the origin and absorb the incoming radiation of the following pulses with the cross section of absorption $\xi^{\prime}\left(\mathrm{cm}^{2}\right)$, which is in general different from $\xi$.

During the pause between two pulses, end links recombine and the $\mathrm{C}=\mathrm{C}$ double bonds are formed with the density $n_{\mathrm{DB}}\left(\mathrm{cm}^{-3}\right)$.

The absorption of the radiation in the material is then described as follows:

$$
\begin{aligned}
\frac{d F^{(i)}(z)}{d z}= & \sum_{k \in\{1,3\}}-\xi_{k}^{(i-1)}(z) F_{k}^{(i)}(z) n_{\mathrm{SB}, f}^{(i-1)}(z) \\
& -\xi_{k}^{(i-1)}(z) F_{k}^{(i)}(z) n_{\text {frag }, f}^{(i-1)}(z),
\end{aligned}
$$

where numbers 1 and 3 attributed to index $k$ denote $1 \omega$ and $3 \omega$ radiation, lower index $f$ designates the final state after the $i$ th pulse-pause cycle in the sequence, while $i$ is given by the upper index in parenthesis. Solving Eq. (3) we obtain the photon flux (photons $/ \mathrm{cm}^{2}$ ) as a function of depth $z$ :

$$
\begin{aligned}
F^{(i)}(z)= & \sum_{k \in\{1,3\}} F_{k}^{(i)}(0) \exp \left\{\int _ { 0 } ^ { z } \left[-\xi_{k}^{(i-1)}(x) n_{\mathrm{SB}}^{(i-1)}(x)\right.\right. \\
& \left.\left.-\xi_{k}^{(i-1)}(x) n_{\text {frag }}^{(i-1)}(x)\right] d x\right\} .
\end{aligned}
$$

The cross section $\xi$ is proposed to decrease with the increasing density of double bonds as follows [24,25]:

$$
\xi_{k}(z)=\frac{\sigma_{\mathrm{ABS}, k}}{1+\frac{n_{\mathrm{DB}}(z)}{\tilde{n}_{\mathrm{DB}, k}}}
$$

Here, $\sigma_{\mathrm{ABS}}\left(\mathrm{cm}^{2}\right)$ and $n_{\mathrm{DB}}\left(\mathrm{cm}^{-3}\right)$ stand for the coefficient of absorption in PMMA, the local density of double bonds, and a fitting parameter for the given index $i$, respectively. $\tilde{n}_{\mathrm{DB}}\left(\mathrm{cm}^{-3}\right)$ is a fitting parameter allowing us to find the optimal slope of the decrease in $\xi(z)$ to fit the experimental data.

Since LCLS pulses are very short (100 fs), cross sections of absorption $\xi$ and $\xi^{\prime}$ are assumed to be constant in time over the pulse-duration period. Effects of cross-linking and other relaxations are neglected for this short time as well. Assuming this, the total density of end links at the end of $i$ th laser pulse is

$$
n_{B}^{(i)}=n_{B, f}^{(i-1)}+\sum_{k \in\{1,3\}} \xi_{k}^{(i-1)} F_{k}^{(i)} n_{\mathrm{SB}, f}^{(i-1)} .
$$

Since the pause between two pulses (more than $30 \mathrm{~ms}$ ) is many orders of magnitude longer than the characteristic times of relaxation and cross-linking, one can assume that all the free end links recombine within this period. We further assume all the end links to form the double bonds at a half density.

Finally, we can write the kinetic equations for the densities and initial conditions at the end of the $i$ th sequence as follows:

$$
\begin{gathered}
n_{B, f}^{(i)} \approx 0 \quad n_{0}=\frac{\rho_{\mathrm{PMMA}} N_{A}}{M_{\mathrm{PMMA}}} \\
n_{\mathrm{DB}, f}^{(i)}=n_{\mathrm{DB}, f}^{(i-1)}+\sum_{k \in\{1,3\}} \xi_{k}^{(i-1)} F_{k}^{(i)} n_{\mathrm{SB}, f}^{(i-1)} \quad n_{\mathrm{SB}, f}^{(0)}=n_{0}, \\
n_{\mathrm{SB}, f}^{(i)}=n_{\mathrm{SB}, f}^{(i-1)}-2 a \sum_{k \in\{1,3\}} \xi_{k}^{(i-1)} F_{k}^{(i)} n_{\mathrm{SB}, f}^{(i-1)} \quad n_{\mathrm{DB}, f}^{(0)}=0, \\
n_{\mathrm{FRAG}, f}^{(i)}=n_{\mathrm{FRAG}, f}^{(i-1)}+2 \sum_{k \in\{1,3\}} \xi_{k}^{(i-1)} F_{k}^{(i)} n_{\mathrm{SB}, f}^{(i-1)} \quad n_{\mathrm{FRAG}, f}^{(0)}=0,
\end{gathered}
$$

where $\rho_{\text {PMMA }}\left(\mathrm{g} / \mathrm{cm}^{3}\right), M_{\text {PMMA }}(\mathrm{g} / \mathrm{mol})$, and $N_{A}$ denote density and molar mass of PMMA and Avogadro's constant, respectively. All the densities are to be computed step by step over all the pulses in the sequence.

The total depth of the desorbed crater is evaluated at the end of the sequence. Here it is defined as a depth where the density of intact molecules is exactly one half of the one in the pristine material:

$$
n_{\mathrm{SB}, f}^{(i)}\left(d_{\text {crater }}\right)=\frac{n_{0}}{2} .
$$

\section{B. Model B}

We develop another mathematical model (below referred to as model B) of the subthreshold damage to PMMA reflecting the statistical nature of all the involved processes.

Now we define the material removal efficiency $\eta$ as a fraction of volume, which desorbs after absorbing a certain dose of energy $\varepsilon$ delivered into the material with a single shot. Function $\eta(\varepsilon)$ was investigated earlier [11] and was found to follow the trend shown in Fig. 1. Under subthreshold irradiation conditions, the efficiency is found to increase gradually in a linear way with a slope $\kappa_{0}^{\text {des }}$ $\left(\mu \mathrm{m}^{3} / \mu \mathrm{J}\right)$ until the absorbed dose reaches a certain value $\varepsilon_{D}$ $\left(\mu \mathrm{J} / \mu \mathrm{m}^{3}\right)$. Exceeding this limit results in a steep increase in efficiency up to unity - the ablation threshold $\varepsilon_{A}\left(\mu \mathrm{J} / \mu \mathrm{m}^{3}\right)$ is reached and for all the higher doses the ablation dominates the material removal process. In Fig. 1 absolute values resulting from referred models are added in order to provide qualitative insight into the phenomena.

As in the previous model, in the case of a sequence of accumulated shots the efficiency is assumed to decrease with the deposited dose. We introduce this phenomenon 


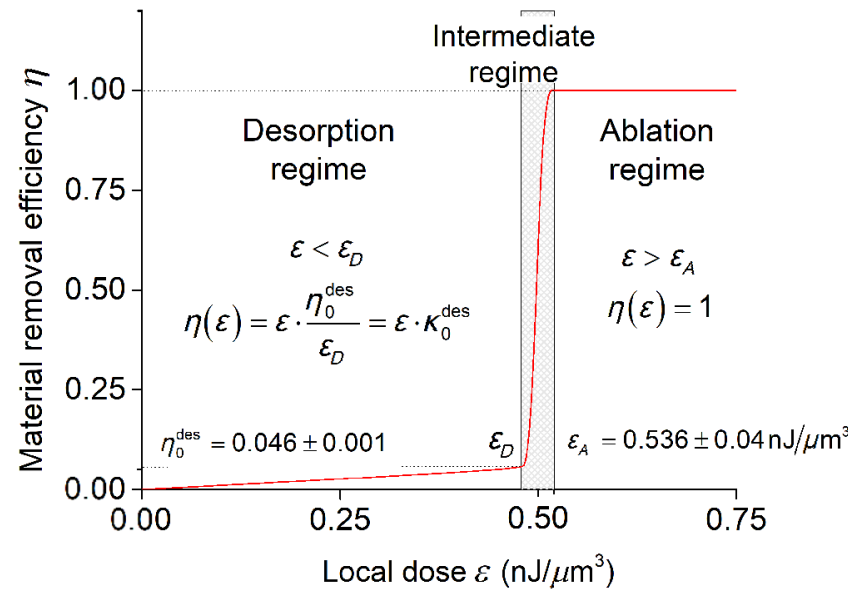

FIG. 1. Schematics of the dependence of material removal efficiency on the local dose absorbed by PMMA; more details on the model can be found in Ref. [11].

into the simulations with an exponential saturation of $\kappa_{0}^{\text {des: }}$ :

$$
\begin{aligned}
& \kappa^{\operatorname{des}}(\varepsilon)=\kappa_{0}^{\operatorname{des}}\left[1-\Delta\left(1-e^{-\gamma \varepsilon}\right)\right], \\
& \kappa^{\operatorname{des}}(0)=\kappa_{0}^{\operatorname{des}}, \\
& \kappa^{\operatorname{des}}(\infty)=\kappa_{0}^{\operatorname{des}}(1-\Delta),
\end{aligned}
$$

where $\gamma\left(\mu \mathrm{m}^{3} / \mu \mathrm{J}\right)$ is the reciprocal value of characteristic dose and $\Delta$ describes the difference in slope between an intact and a fully saturated material, i.e., intact PMMA molecules and molecules containing only double bonds in the main chain.

The accumulated dose in a certain depth is defined as a sum of doses delivered by individual shots:

$$
D_{i}(z)=\sum_{j=1}^{i} \varepsilon_{j}(z)
$$

And the corresponding slope of desorption efficiency for a subsequent shot is then given by the equation:

$\eta_{i}^{\mathrm{des}}(z)=\kappa_{i-1}^{\mathrm{des}}(z) \varepsilon_{i}(z)=\kappa_{0}^{\mathrm{des}}\left\{1-\Delta\left[1-e^{-\gamma D_{i-1}(z)}\right]\right\} \varepsilon_{i}(z)$.

Since the experiment is carried out under the ultrahigh vacuum conditions, we assume the liberated fragments to escape entirely from the irradiated surface, which implies no further absorption of the radiation by these fragments.

By integration of Eq. (11) along the whole beam path inside the material we obtain the overall decrease in the linear mass density. This is assumed to be compensated by collapse of the mass, which results in evolution of the crater in the beam direction.

For the evaluation purpose we assume that the surface of the material moves in the beam direction $[d(\mu \mathrm{m})$ grows up, thinning of the material] after each shot, whereas the distribution of the deposited dose remains the same, i.e., each shot causes desorption of a surface layer with a thickness given by the integral of Eq. (11). The idea of this process is depicted in Fig. 2.

As in the previous model we assume the simultaneous action of the $1 \omega$ and $3 \omega$ component and a distribution of the dose inside the material in accordance with the Lambert-Beer law. Taking into account the effect of moving surface, one can write the equations for the single shot and accumulated dose at the depth $z$ as follows:

$$
\begin{aligned}
& \varepsilon_{i}(z)=\left[\varepsilon_{i 10} e^{\frac{-\left(z-d_{i-1}\right)}{l_{\text {att1 }}}}+\varepsilon_{i 30} e^{\frac{-\left(z-d_{i-1}\right)}{l_{\text {att3 }}}}\right] \Theta\left(z-d_{i-1}\right), \\
& D_{i}(z)=\sum_{j=1}^{i} \varepsilon_{j}(z)=\sum_{j=1}^{i}\left[\varepsilon_{j 10} e^{\frac{-\left(z-d_{j-1}\right)}{l_{\text {attl }}}}+\varepsilon_{j 30} e^{\frac{-\left(z-d_{j-1}\right)}{l_{\text {att3 }}}}\right] \Theta\left(z-d_{i-1}\right),
\end{aligned}
$$

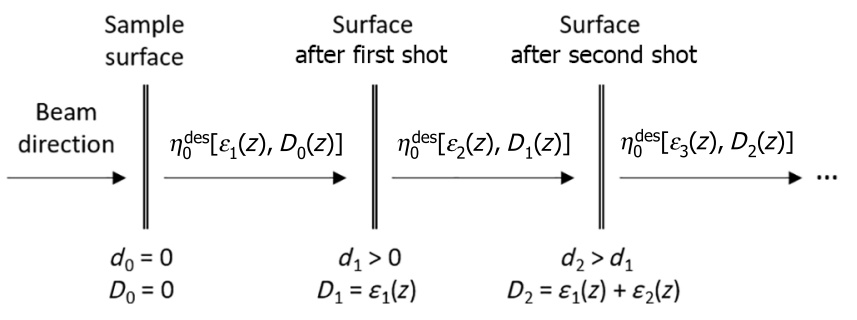

FIG. 2. Schematic representation of the sample surface shift during the irradiation sequence together with corresponding constants used for the evaluation of the maximum crater depth after each individual shot interaction. where $\varepsilon_{i k 0}\left(\mu \mathrm{J} / \mu \mathrm{m}^{3}\right)$ denotes a peak absorbed dose of $i$ th pulse, index $k$ distinguishes between the fundamental and $3 \omega$ harmonic component, $l_{\text {att }}(\mu \mathrm{m})$ stands for the photon attenuation length in PMMA, and $\Theta(x)$ is the Heaviside step function (its value is 0 for $x<0$ and 1 for $x>0$ ). Peak dose is defined as the peak fluence divided by the attenuation length, i.e.,

$$
\varepsilon_{0}=\frac{E_{P}}{A_{\text {eff }} l_{\text {att }}},
$$


where $E_{P}(\mu \mathrm{J})$ and $A_{\text {eff }}\left(\mu \mathrm{m}^{2}\right)$ denote pulse energy and effective area of the laser beam.

A shift in the surface location induced by the $i$ th individual shot in irradiation sequence is then

$$
\begin{aligned}
\Delta_{i} & =d_{i}-d_{i-1}=\int_{0}^{\infty} \eta_{i}^{\mathrm{des}}(z) d z \\
& =\int_{d_{i-1}}^{\infty}\left\{\kappa_{0}^{\mathrm{des}}-\kappa_{0}^{\mathrm{des}} \Delta\left[1-e^{-\gamma D_{i-1}(z)}\right]\right\}
\end{aligned}
$$

$$
\begin{aligned}
& \times\left[\varepsilon_{i 10} e^{\frac{-\left(z-d_{i-1}\right)}{l_{\text {attl }}}}+\varepsilon_{i 30} e^{\frac{-\left(z-d_{i-1}\right)}{l_{\text {att3 }}}}\right] d z, \\
\equiv & \Delta_{i}^{D}+\Delta_{i}^{H} .
\end{aligned}
$$

As indicated in Eq. (14), the change in depth can be expressed as a contribution from the pure desorption $\Delta_{i}^{D}$ reduced by the term reflecting the material hardening $\Delta_{i}^{H}$. By the definition both the terms must fulfil the inequality: $\Delta_{i}^{H} \leq 0 \leq\left|\Delta_{i}^{H}\right| \leq \Delta_{i}^{D}$.

After integration we find the final forms of these terms:

$$
\begin{aligned}
& \Delta_{i}^{D}=\int_{d_{i-1}}^{\infty} \kappa_{0}^{\operatorname{des}}\left[\varepsilon_{i 10} e^{\frac{-\left(z-d_{i-1}\right)}{l_{\text {att }}}}+\varepsilon_{i 30} e^{\frac{-\left(z-d_{i-1}\right)}{l_{\text {att3 }}}}\right] d z=l_{\text {att } 1} \kappa_{0}^{\text {des }} \varepsilon_{i 10}+l_{\text {att } 3} \kappa_{0}^{\text {des }} \varepsilon_{i 30}, \\
& \Delta_{i}^{H}=-\int_{d_{i-1}}^{\infty} \kappa_{0}^{\mathrm{des}} \Delta\left[1-e^{-\gamma D_{i-1}(z)}\right]\left[\varepsilon_{i 10} e^{\frac{-\left(z-d_{i-1}\right)}{l_{\text {att1 }}}}+\varepsilon_{i 30} e^{\frac{-\left(z-d_{i-1}\right)}{l_{\text {att3 }}}}\right] d z, \\
& =-\kappa_{0}^{\mathrm{des}} \Delta\left(l_{\mathrm{att1}} \varepsilon_{i 10}+l_{\text {att } 3} \varepsilon_{i 30}\right)+\kappa_{0}^{\mathrm{des}} \Delta l_{\mathrm{att1}} \varepsilon_{i 10} \frac{e^{\frac{d_{i-1}}{l_{\text {attl }}}-\Gamma_{i 3}-\Gamma_{i 1} e^{-\frac{d_{i-1}}{l_{\text {att1 }}}}}\left(e^{\Gamma_{i 1} e^{-\frac{d_{i-1}}{l_{\text {att }}}}}-1\right)}{\Gamma_{i 1}}
\end{aligned}
$$

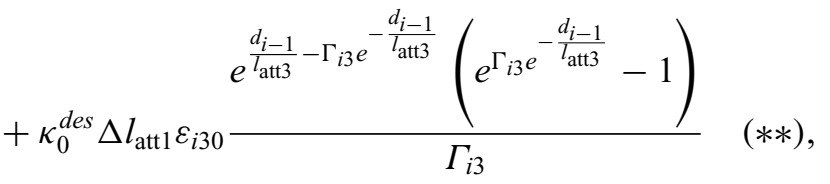

where

$$
\Gamma_{i 1}=\gamma \sum_{j=1}^{i-1} \varepsilon_{j 10} e^{\frac{d_{j-1}}{l_{\text {att }}}} \quad \Gamma_{i 3}=\gamma \sum_{j=1}^{i-1} \varepsilon_{j 30} e^{\frac{d_{j-1}}{l_{\text {att } 3}}}
$$

In the evaluation of the integral in Eq. (15), two approximations are made in order to find an analytical solution. (i) Within the layer close to the surface the amplitude of $3 \omega$ radiation is considered a "constant background" due to its very long attenuation length $(39.71 \mu \mathrm{m})$ compared to the assumed thickness of the layer. This applies to the term marked with $(*)$ and we set

$$
e^{-\frac{z-d_{i}}{l_{\text {att3 }}}} \approx 1
$$

The other approximation (ii) is made for the deep layer where the fundamental component is already very weak and can be neglected. This takes place during evaluation of the term marked with $(* *)$ where we set

$$
e^{-\frac{z-d_{i}}{l_{\text {att }}}} \approx 0 .
$$

The final depth of the crater eroded by the shot sequence is defined as a simple sum of contributions from the individual shots $\Delta_{i}$.

\section{RESULTS AND DISCUSSION}

An AFM image of the ablation crater induced by a single $830-\mathrm{eV}$ laser shot is shown in Fig. 3. The shown sample is irradiated at the fluence three times exceeding the ablation threshold. We identify the damage threshold fluence for the used photon energy in a series of measurements as follows. The dependence of a damaged area of an irradiated PMMA surface on a logarithm of the laser pulse energy $E_{\text {pulse }}(\mathrm{mJ})$ is displayed in the inset in Fig. 4. The intersection of the fitted line with the $x$ axis gives the threshold energy $E_{\mathrm{th}}(\mathrm{mJ})$, i.e., the maximal pulse energy at which the ablated surface area is equal to zero. The F-scan technique [26] is used to 


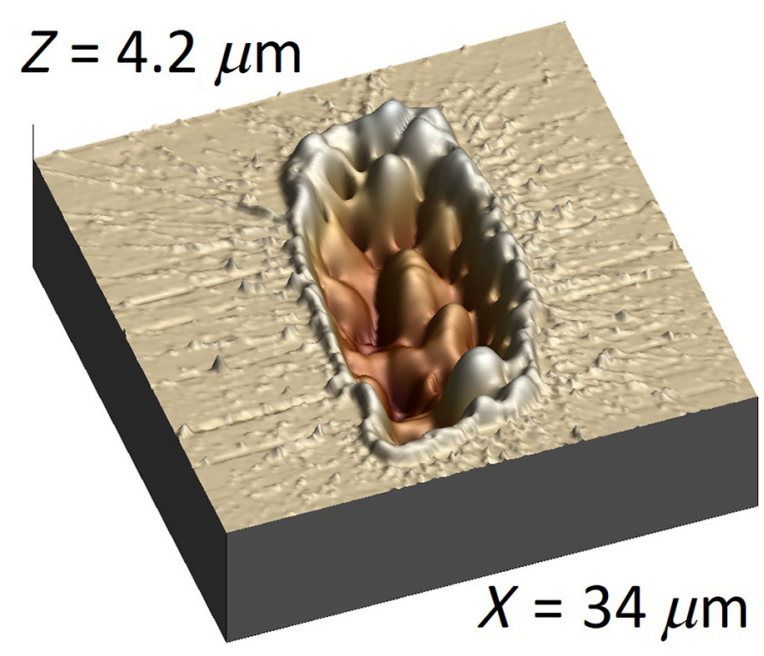

FIG. 3. AFM image of PMMA irradiated with a single LCLS shot at a fluence well above the ablation threshold $\left(F=3 F_{\text {th }}\right)$.

determine the effective area $A_{\text {eff }}\left(\mu \mathrm{m}^{2}\right)$ of the focal spot as shown in Fig. 4. The ratio between the laser pulse energy and the threshold energy is plotted here against the area of the surface ablated by this pulse. The dataset is fitted with the double-Gaussian model [27]. Finally, the effective area is given by the area under this curve - it is found to be equal to $131.6 \pm 4.5 \mu \mathrm{m}^{2}$. Then, a ratio between the threshold energy and the effective area gives a threshold fluence of $99.7 \pm 7.4 \mathrm{~mJ} / \mathrm{cm}^{2}$ for the single-shot exposure.

Figure 5 shows that the damage induced by multiple shots on the sample surface with a fluence below

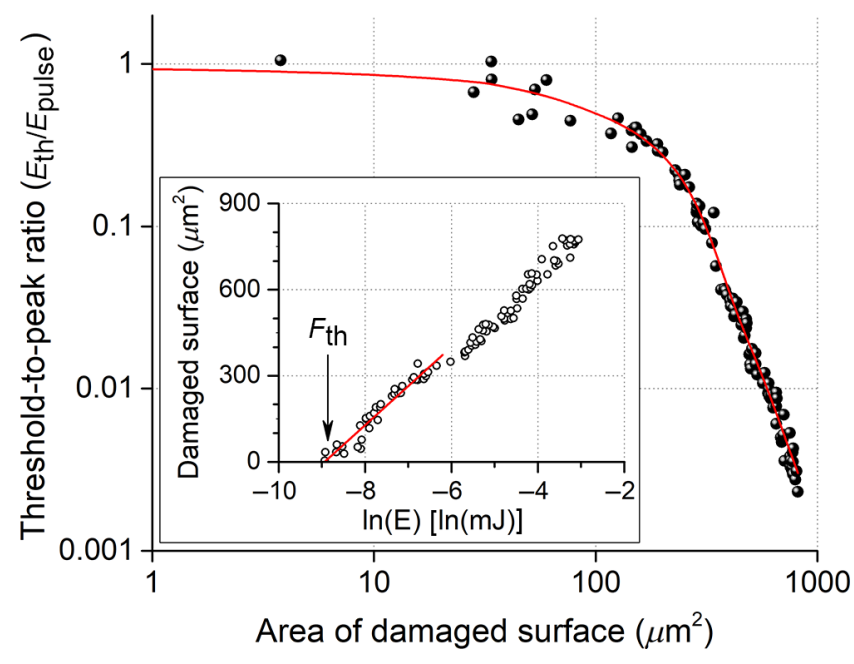

FIG. 4. Fit of the threshold-to-peak ratio with doubleGaussian model. Area under the fitting curve is equal to the effective area of the beam $\left(131.6 \pm 4.5 \mu \mathrm{m}^{2}\right)$. Inset: areas of PMMA surface damaged by a single shot of $830-\mathrm{eV}$ laser radiation as a function of the pulse energy. It shows clearly that ablation of PMMA by a single shot begins at $F_{\mathrm{th}}=99.7 \mathrm{~mJ} / \mathrm{cm}^{2}$.
$99.7 \mathrm{~mJ} / \mathrm{cm}^{2}$ is very different from that caused by a single shot at higher fluence (cf. Fig. 3). The subthreshold damage is much cleaner, smoother, without any material extrusions, molten zones, etc. An average surface roughness is also lower than in a case of above-threshold irradiation.

Earlier, a simple model is developed to describe a nonlinear desorption mechanism in PMMA under weak irradiation with short-wavelength coherent source, which is successfully used for high-order harmonic XUV source [24,25]. According to the model, a nonlinear dependence of the crater depth on the accumulated dose (energy deposited per unit of mass $\mathrm{J} / \mathrm{kg}$ ) is expected even for LCLS radiation. To investigate such a dependence experimentally, three sets of craters are eroded on the PMMA surface at a fluence well below the single-shot ablation threshold: $0.4,4$, and $40 \%$ of the threshold value (all these values are related to the fluence of the $1 \omega$ component). Each of these sets consists of craters made with a different number of accumulated shots. Figures 6 and 7 summarize dependences of maximal crater depths on the number of accumulated shots and on the total deposited dose, respectively.

Two kinds of nonlinearity can be distinguished in Figs. 6 and 7. The slope of the curves shows that the desorption etch rate is decreasing with an increase of the total dose. This phenomenon can be attributed to a growing density of $\mathrm{C}=\mathrm{C}$ double bonds formed by cross-linking in the irradiated PMMA [28-31]. The mechanism of such a structure-making process is depicted in Fig. 8. The crosslinking enhances the radiation hardness of the material (i.e., the binding energy of $\mathrm{C}=\mathrm{C}$ double bond is higher than for $\mathrm{C}-\mathrm{C}$ single bond), increases the average molecular weight of PMMA chains and reduces the mobility of low-molecular fragments in the irradiated material. In contrast, a major part of the incident radiation initiates both the main chain scissions, resulting into a depolymerization, and the radiolysis of ester and methyl groups. Both processes create small volatile fragments of a polymer molecule liberated from the irradiated surface into a vacuum. Radiation chemistry of PMMA is reviewed in Refs. [32-35].

The presence of the $\mathrm{C}=\mathrm{C}$ double bonds after irradiation is revealed by the Raman spectroscopy with spatial resolution. Figures 9 and 10 illustrate the chemical changes in the irradiated material after 300 shots at a fluence level of $40 \mathrm{~mJ} / \mathrm{cm}^{2}$. Appearance of a new band in Raman spectrum at $1650 \mathrm{~cm}^{-1}$ provides an evidence of cross-linking induced by $x$ rays.

We use Raman spectroscopy in order to investigate the formation of $\mathrm{C}=\mathrm{C}$ double bonds parallel to the drop in density of methyl and ester groups. The ratios between area of a new band at $1650 \mathrm{~cm}^{-1}$ and bands corresponding to bonds of ester and methyl groups are plotted against a total accumulated dose and are summarized in Fig. 11(a). A rising trend 

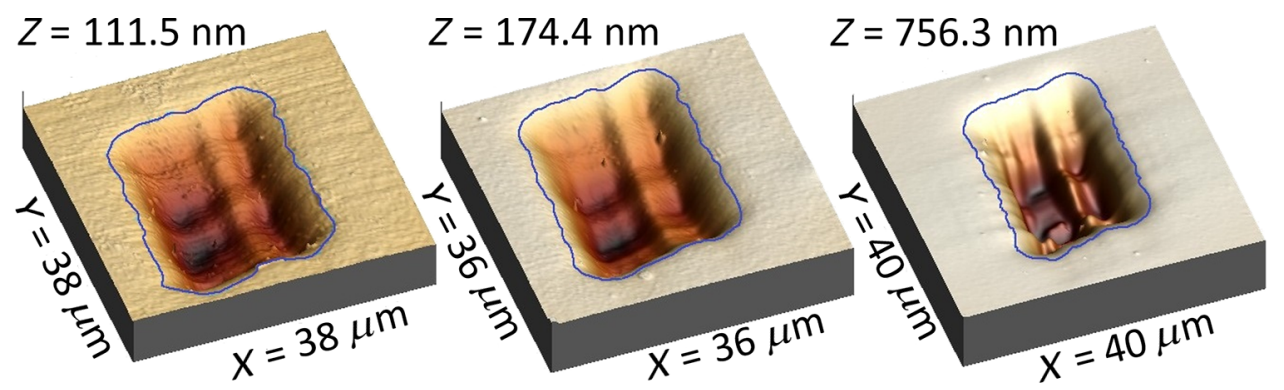

FIG. 5. AFM images of craters eroded in PMMA with 300 accumulated shots of LCLS radiation sent to the surface at three fluence levels: (left) $0.4 \%$, (middle) $4 \%$, and (right) $40 \%$ of the single-shot ablation threshold. Areas taken for the roughness evaluation are circumscribed with a blue line. in all the dependences is evidence of the increasing density of the double bonds. As shown in Fig. 11(b), a slight increase in ratios $\mathrm{S}\left(1125 \mathrm{~cm}^{-1}\right) / \mathrm{S}\left(800 \mathrm{~cm}^{-1}\right)$ and $\mathrm{S}\left(1125 \mathrm{~cm}^{-1}\right) / \mathrm{S}\left(1450 \mathrm{~cm}^{-1}\right)$ is found, indicating the densities of ester chains and methyl groups drop faster than the one of backbone single bonds. This supports the assumption that these volatile fragments escape the irradiated surface while the rest of the polymer molecule stays.

The material hardening due to the cross-linking seems to be a reverse effect with respect to the incubation effect [36,37]. Although there is a similarity in a reduced etch rate connected with irradiation phases when these effects take place, there is a dramatic difference in mechanisms. Incubation pulses are located at the beginning of the irradiation, when absorption is still low and material fragments remain too large to evaporate. The incubation effect can be explained by an increase of absorption coefficient or structural changes associated with defects produced by first pulses making desorption and ablation by subsequent pulses easier. In contrast to that, the material hardening

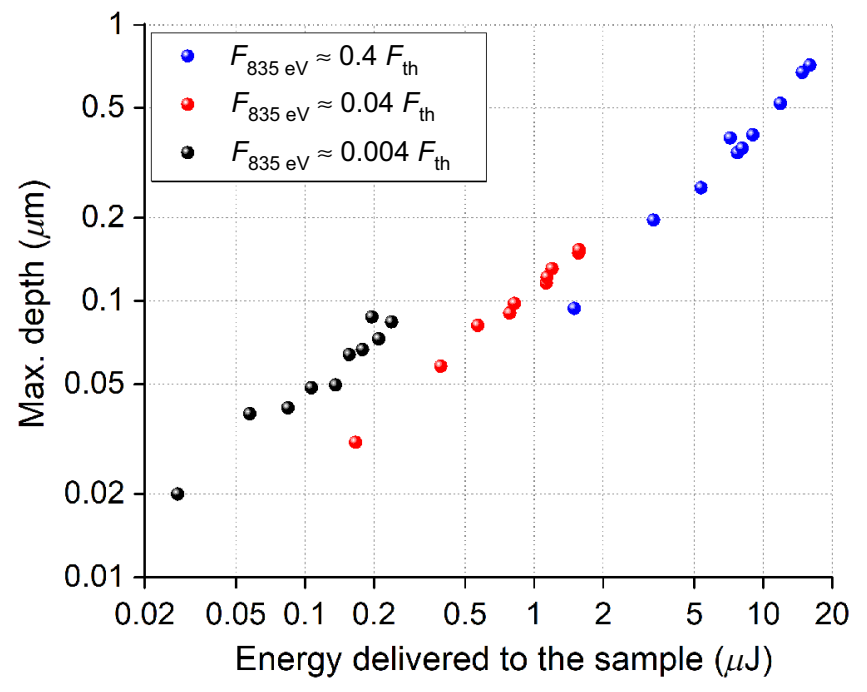

FIG. 6. Dependence of the maximum crater depth on the total X-ray laser energy at three different fluences: (black) $0.4 \%$, (red) $4 \%$, and (blue) $40 \%$ of the ablation threshold. Deposited energy is determined according to an energy content of each particular laser pulse measured by photoionization GMD. Contributions from the $3 \omega$ component are incorporated as well. occurs during the whole irradiation course. It reduces an etch rate due to radiation-induced cross-linking of polymer molecules, which leads to an increase of molecular weight of fragments lowering its evaporation probability.

The nonlinearity of the second kind comes from comparing the depths of craters corresponding to the same value of accumulated dose but to a different fluence at which they are formed. It is clearly visible in Fig. 6 that a crater created at a lower fluence level is deeper than the one formed at a higher fluence level.

This can just be explained with presence of $3 \omega$ radiation in the incoming pulses. As the transmission of the gas attenuator is not constant for both $1 \omega$ and $3 \omega$ components the ratio between their contributions to the total accumulated dose varies with each different pressure setting. The lower transmission is set for fundamental photon energy the more the relative $3 \omega$ content grows. This nonlinearity disappears once the maximal depth of the crater is plotted against the local dose accumulated into the PMMA with both spectral components, as shown in Fig. 7.

The dose-rate effects could, in principle, result from an influence of the energy dissipation via heating. Although

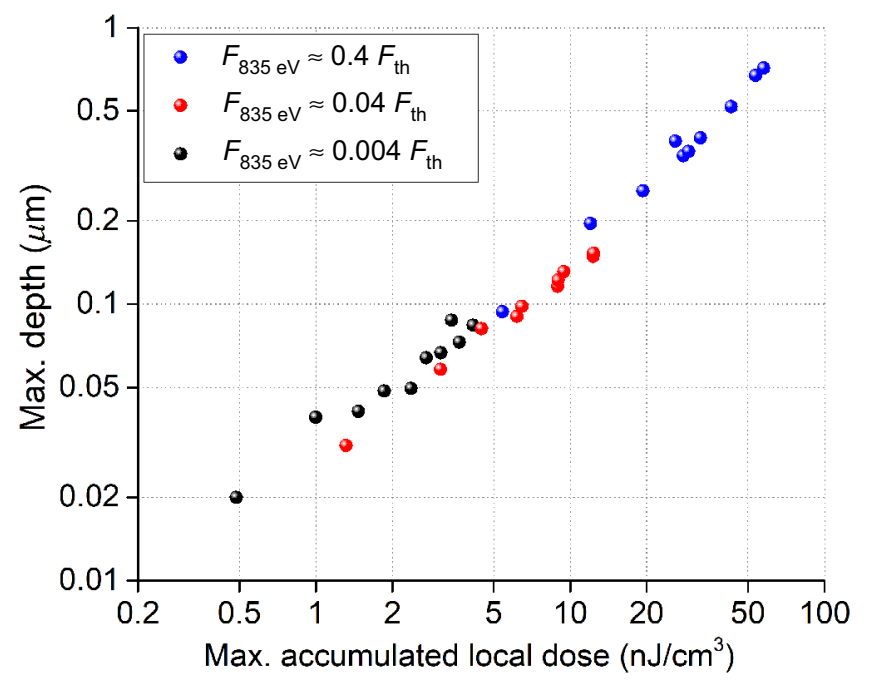

FIG. 7. Dependence of the maximum crater depth on the total accumulated dose from both the $1 \omega$ and $3 \omega$ spectral component at three different fluences: (black) $0.4 \%$, (red) $4 \%$, and (blue) $40 \%$ of the ablation threshold. 
(a)<smiles>COC(=O)C(C)(C)CC(C)(CC(C)(C)C(=O)OC)C(=O)OC</smiles>

(c)<smiles>COC(=O)C(C)(C)CC(C)(C)C</smiles>

(b)

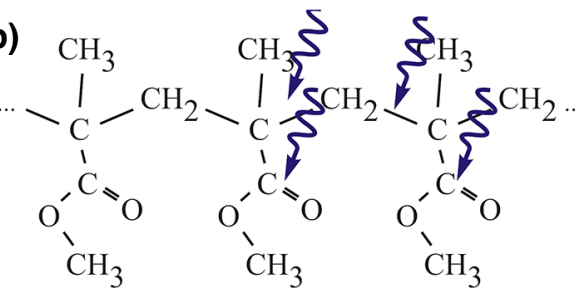

(d)<smiles>COC(=O)C(C)(C)CC(C)=C(C)C</smiles>

FIG. 8. Reaction schemes of a $\mathrm{C}=\mathrm{C}$ bond formation in a PMMA molecule: (a) intact PMMA chain, (b) chain-scission effect caused by incident radiation, (c) and (d) forming the double bond via the cross-linking effect; according to Refs. [28,29]. radiation chemical processes do not depend on the temperature dramatically, the transport processes usually exhibit a very strong temperature dependence. So, at a higher fluence we may expect a higher heating rate reaching a higher temperature at the surface and in the near-surface layer. The heat enhances a transport of low-molecular fragments toward the surface and then their desorption from the surface into vacuum [see, for example, our earlier results obtained in polyterafluoroethylene (PTFE) [38] exposed at various temperatures to short pulses of XUV radiation emitted by laser-produced plasmas].

There are two reasons for making the conclusion that our results are not heat influences. First, the etch rate decreases at a higher dose-rate level (see Figs. 6 and 7) instead of increasing, so the heating itself cannot be the reason of the observed behavior. Second, PMMA irradiated by ionizing radiation at elevated temperatures exhibits

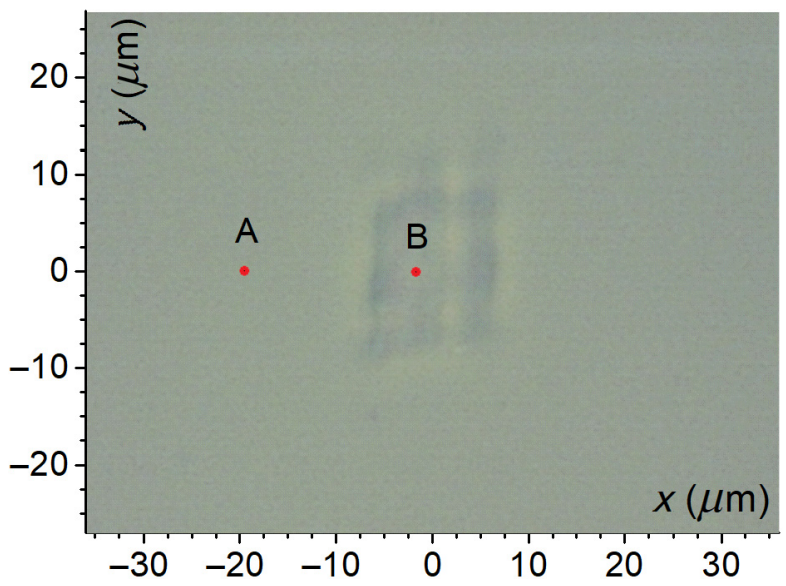

FIG. 9. Positions on the PMMA surface where the Raman spectra are acquired from (a) unirradiated area and (b) area irradiated with 300 shots at fluence level of $40 \%$ of the ablation threshold. a specific change in its morphology due to the formation of gaseous radiolytic products, see, for example, Refs. [32-35]. Therefore, the roughness of the inner surface of LCLS-etched crater should increase at higher LCLS dose rates if such processes play a noticeable role. To check this possibility, the RMS roughness of the irradiated surface is measured by the AFM probe inside the craters produced by 300 accumulated shots (Fig. 5). The values are found to be $2.1,2.4$, and $1.95 \mathrm{~nm}$ for fluence levels of $0.4,4$, and $40 \%$ of single-shot ablation threshold, respectively. These three values are even lower than the RMS roughness found on the unirradiated pristine PMMA surface, which is equal to $2.6 \mathrm{~nm}$. The radiation smoothening is important not only for understanding the erosion mechanisms but the finding seems to be promising for

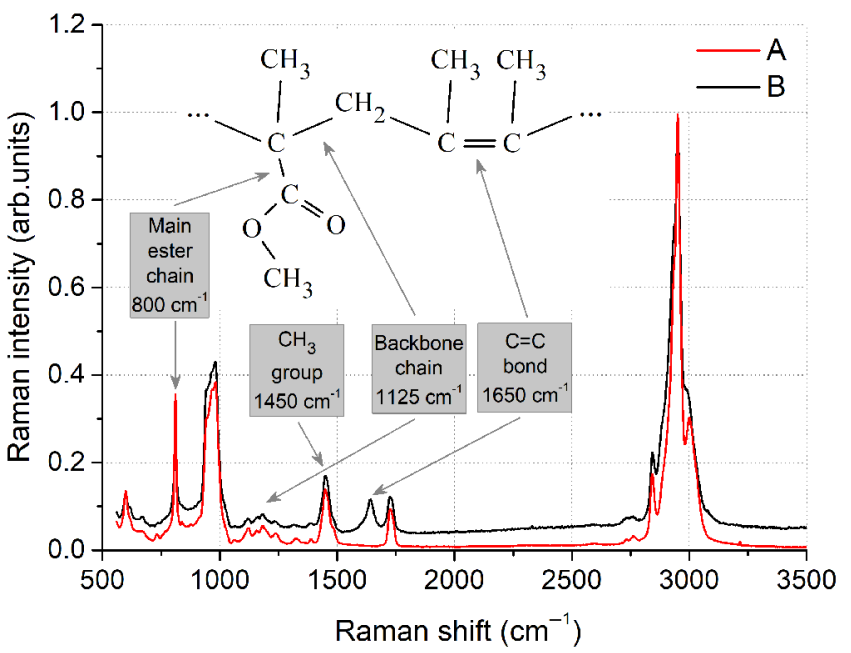

FIG. 10. Raman spectra of PMMA corresponding to the points of measurements depicted in Fig. 7. A new band at $1650 \mathrm{~cm}^{-1}$ formed due to irradiation is assigned to $\mathrm{C}=\mathrm{C}$ bond associated with the radiation-initiated cross-linking in PMMA. 

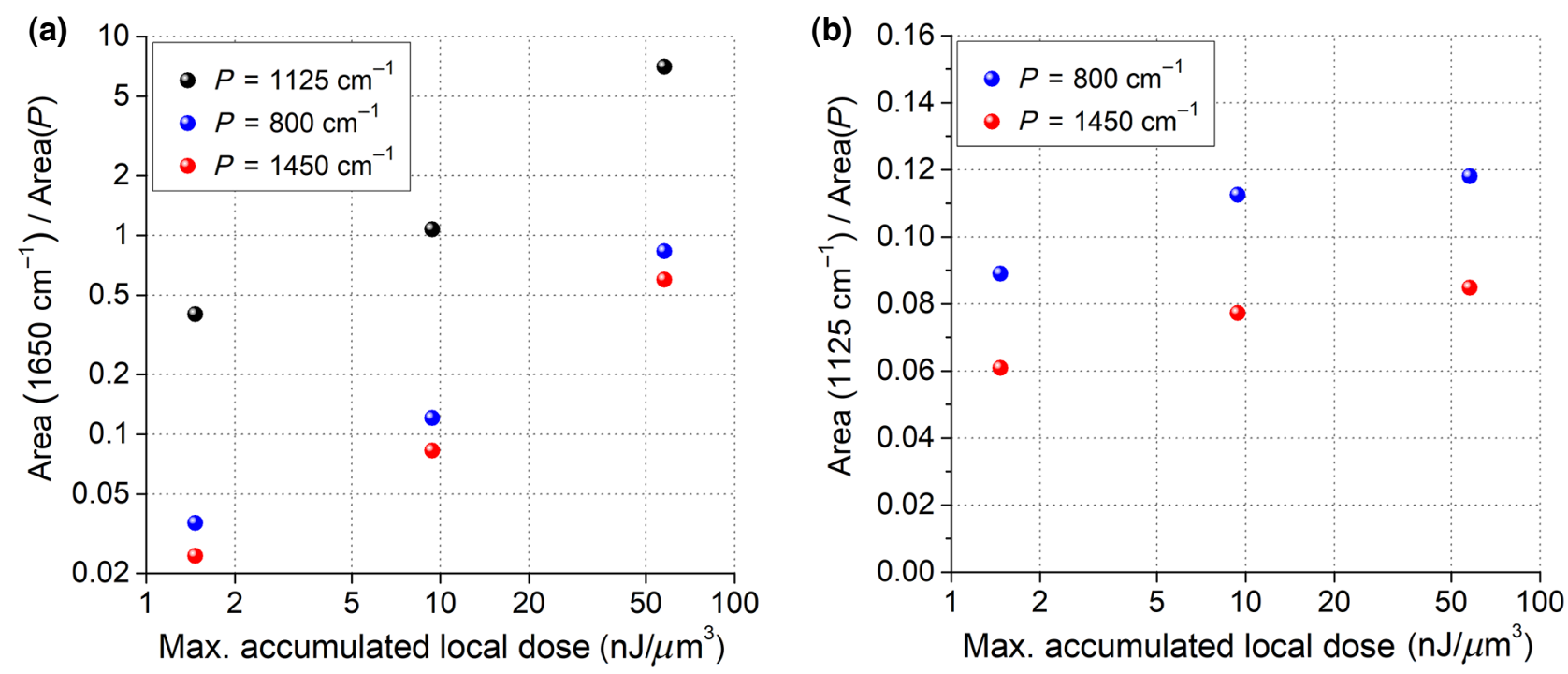

FIG. 11. (a) Dependence of the area ratios of the new band at $1650 \mathrm{~cm}^{-1}$ corresponding to formed $\mathrm{C}=\mathrm{C}$ double bonds to bands assigned to backbone chains $\left(1125 \mathrm{~cm}^{-1}\right)$ and ester $\left(800 \mathrm{~cm}^{-1}\right)$ and methyl $\left(1450 \mathrm{~cm}^{-1}\right)$ groups on accumulated doses; (b) an increase of the ratio of the backbone chain signal to bands belonging to ester (blue) and methyl (red) groups with increasing accumulated dose.

prospective utilization of compact XUV FELs in materials patterning (see, for example, Ref. [39]).

In general, the ratio of thermal and nonthermal processes following absorption of energetic photons is primarily given by a constitution of irradiated material and its properties defined by the constitution. A radiation resistance of the material plays the key role. Thus, in PMMA — which can be easily decomposed by ionizing radiation with a high radiation chemical yield (for a review see Refs. [34,35]) - we may expect a minor portion of deposited energy to be dissipated, converted into heat, while a major part of energy causes ionizations, excitations, and subsequently chemical reactions and nonthermal phase transitions. In contrast to that, in radiation-resistant inorganic materials, e.g., monocrystalline silicon, a significant portion of deposited energy should be released as heat because there are no other channels opened at a microscopic level through which deposited energy can flow. The absence of thermal depolymerization features on irradiated PMMA surfaces (see Fig. 5) and the character of an intensity dependence of etch rate (Figs. 6 and 7) testify to a fully nonthermal character of the desorption processes initiated under these irradiation conditions, i.e., ultrashort pulses of high-energy photons delivered to the sample at a relatively low repetition rate.

Coulomb repulsion of positively charged fragments can also influence the etch rate at different fluence levels. When photoelectrons and secondary electrons leave the surface, an intermolecular interaction of remaining cation radicals may enhance the desorption processes. Earlier, we observed such behavior in FEL-illuminated fullerene solids [40]. However, this also results in a higher etch rate at an elevated dose rate, contradictory to the observation seen in Figs. 6 and 7. Therefore, any noticeable participation of such processes in the PMMA erosion under the irradiation conditions reported in this paper is unlikely.

We use both the above-mentioned models, A and B, to fit the experimental data. The ratio between $1 \omega$ and $3 \omega$ component in full-power beam is assumed to be $1 / 100$. Appropriate beamline transmission and the attenuation factor of a gas attenuator are taken into account for both $1 \omega$ and $3 \omega$ components in order to determine the energy ratios $E_{1 \omega} / E_{3 \omega}$ at the sample surface. The last quantity to be determined is effective area of the $3 \omega$ component. It is found through fitting the data with model $\mathrm{B}$, where the $A_{\text {eff }}$ from Eq. (13) is set as a variable fitting parameter. A resulting value of $57.4 \mu \mathrm{m}^{2}$ is then used for all the following simulations.

Results of the simulations are summarized in Fig. 12. The maximal crater depth is plotted here against the maximum local dose of energy deposited in the PMMA sample, i.e., sum of contributions both from $1 \omega$ and $3 \omega$ components. A contribution of the third harmonic to the subthreshold erosion processes should be considered. Agreement of experimental data and computer simulation is not reached if the $3 \omega$ contribution is ignored.

Both models fit the measured data very well especially in the case of a high number of pulses accumulated. Although model A is developed for desorption at very low etch rates and high repetition rates $(3.5 \mathrm{pm}$ per laser pulse at $1 \mathrm{kHz}[24,25])$, its credibility is verified up to approximately $3 \mathrm{~nm}$ per pulse at $30 \mathrm{~Hz}$ in this work. Surprisingly, such a high etch rate can still be simulated with the model assuming static response of the material 


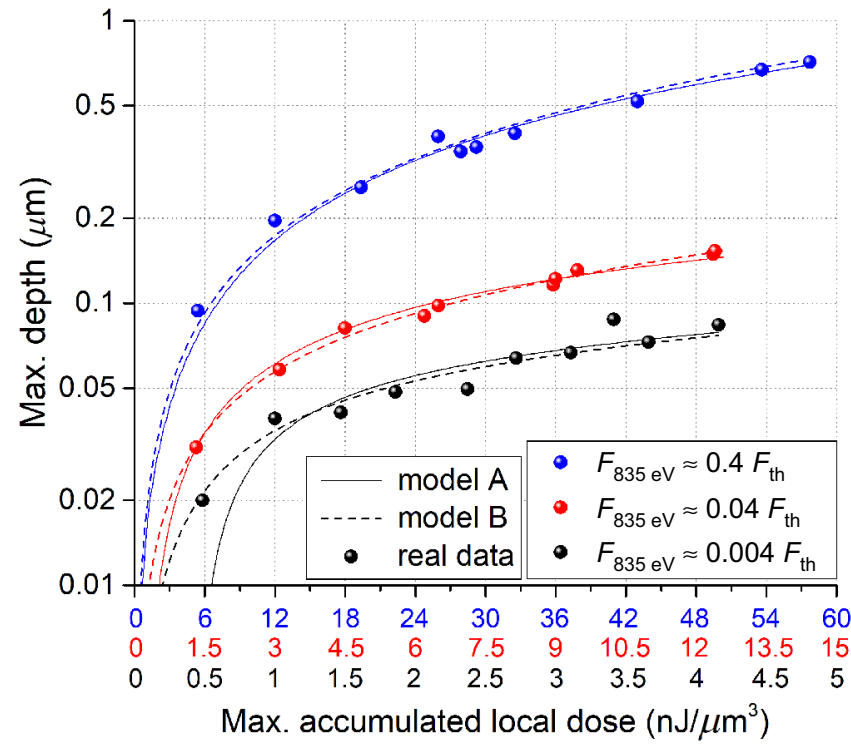

FIG. 12. Maximal depth of craters eroded with accumulated LCLS laser pulses at fluence levels of $40 \%$ (blue), $4 \%$ (red) and $0.4 \%$ (black) of the ablation threshold fitted both with model A (solid line) and B (dashed line).

with no mass removal during the irradiation. However, for highly attenuated beams and low number of accumulated shots we see a discrepancy in the predictions made with model A as shown in Fig. 13. This can be explained by a low initial probability of volatile fragment formation at the beginning of the irradiation process. It comes from the fact that a gradual decomposition of PMMA macromolecules requires more shots for reaching the boundary condition to start an efficient desorption process leading to a crater appearance. Hence, model A is more suitable for simulation of long irradiation series at a wide range of fluences, providing the idea about the chemical alterations in the whole volume of the sample. Such a procedure is limited by higher requirements on time and computation performance. The densities of intact molecules, double bonds, and fragments are to be recursively evaluated for each individual shot in a high number of depth levels within every fitting iteration.

Based on the results, model B provides good agreement with the experimental data both for a wide range of fluences and an arbitrary number of accumulated shots, including irradiation series with only a few accumulated shots. Fitting the datasets is considerably faster than in the previous case since the final crater depth corresponds to the sum of analytical functions. By definition this model follows the natural process of crater development, i.e., the surface moves after each laser shot by a certain step leaving the desorbed volume empty. On the other hand, some aspects are still to be improved in the future, e.g., involving the term of backward recombination of the end links with neither double-bond formation nor fragment escape,

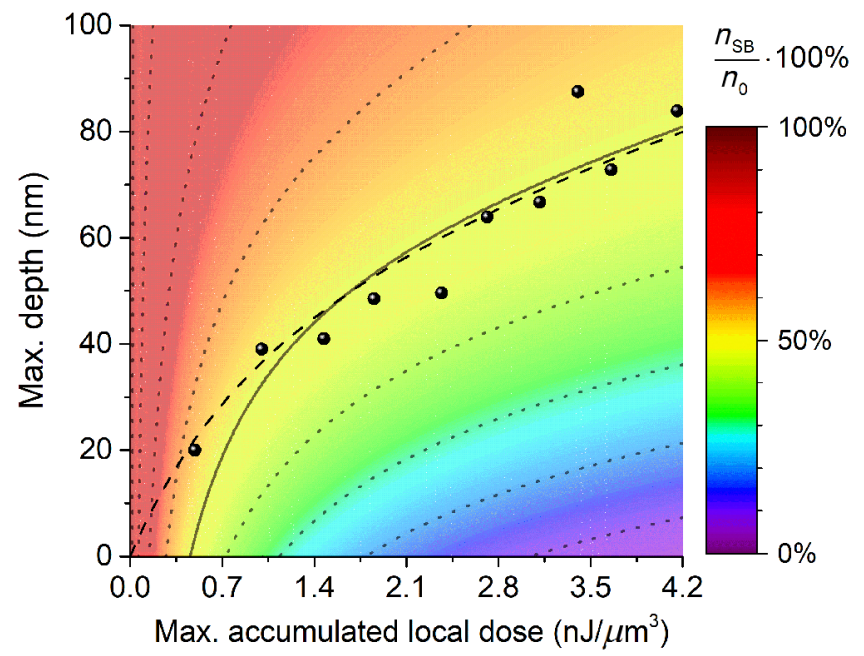

FIG. 13. Maximal depth of craters eroded with LCLS laser pulses at fluence level of $0.4 \%$ of the single-shot ablation threshold, including predictions made with model A (solid line) and model B (dashed line). Progress in density of intact molecules of PMMA during irradiation sequence simulated with model $\mathrm{A}$ is illustrated by means of color scale. Isodensities (dotted lines) are plotted in $10 \%$ steps of the full scale. Isodensity of $50 \%$ of $n_{\mathrm{SB}} / n_{0}$ refers to the predicted crater depth.

or taking into account the lower probability of fragment liberation from deeper layers.

At the end of this section, let us discuss the practical relevance of the above-summarized experimental and theoretical findings. We may identify technically relevant research and development areas where these findings can be applicable:

(a) The desorption processes treated in the present paper represent the only way of how to imprint the focused beam of short-wavelength laser into low density, low- $Z$ materials [e.g., PMMA, PTFE, poly(butene sulfone)] to reveal lateral and longitudinal intensity distribution and other focused beam characteristics. Conventionally, ablation imprints of x-ray laser beams can only be obtained in heavy, high- $Z$ solids (e.g., $\mathrm{PbI}_{2}$, $\mathrm{PbWO}_{4}, \mathrm{Au}$ ), where an attenuation length is sufficiently short. Their utilization brings some difficulties (narrowed dynamic range, complications due to thermomechanical and phase-transition processes and so on). Therefore, using organic polymers eroded in the desorption mode by many pulses demonstrates a reliable and accurate alternative to the single-shot ablation of refractory inorganic systems, see, for example, Ref. [17]. Choosing an appropriate beam characterization technique is important not only for performing various experiments with x-ray lasers properly but it provides a feedback for further development and optimization of x-ray laser systems and their optics. Although short-wavelength lasers of various kinds are being developed for decades, their nature is not 
yet deeply understood and its technology is not developed so efficiently as long-wavelength (UV-vis-IR) lasers are.

Recently, the advent of high-repetition-rate (quasicw) short-wavelength lasers brings another issue in the field. Adaptation of beam diagnostics for high-repetitionrate regimes of operation represents a demanding task for machine scientists and in-house research and development at most FEL facilities. The heat pile-up effect accompanying the high-repetition-rate exposure is the main limiting factor not only for optical elements - see paragraph (c) below - but also for detectors and imagers, e.g., x ray CCDs, microchannel plates, scintillators, etc. With an advent of MHz FELs, e.g., FLASH II, European XFEL, and LCLS II, this question is nowadays a subject of extensive research. It was demonstrated by Sobierajski et al. [13] that the damage threshold of silicon can be significantly lower in the $\mathrm{MHz}$ regime of operation due to nondiminishing heat accumulation. Similar initial experiments have already been performed with PMMA but using $\mathrm{MHz}$ soft $\mathrm{x}$-ray radiation trains only. Experiments with $\mathrm{MHz}$ pulse trains of $\mathrm{x}$ rays are planned.

(b) Using the $\mathrm{x}$-ray laser-induced desorption for surface nanostructuring could bring at least three advantages:

i. The short wavelength radiation may produce smaller lateral details (diffraction-limited pattern transfer from a mask) and deeply eroded structures (an attenuation length increases with decreasing wavelength), therefore, a high aspect ratio can be achieved. A direct structuring of PMMA has already been tested with plasma-based soft x-ray lasers $[41,42]$ in ablation mode. Nanopatterning experiments have been performed with $x$-ray FEL beam in neither ablation nor desorption mode yet.

ii. Since the $\mathrm{x}$-ray desorption is dominated by nonthermal processes, the residual surface cannot be influenced by thermomechanical events like material melting, piston effect, etc. [39]. FEL devices generate ultrashort pulses, which can discern desorption and ablation regimes in PMMA [11] while short pulses provided by plasma-based XUV and $\mathrm{x}$-ray lasers cannot [43].

iii. Because of an extremely low level of backscattered $\mathrm{x}$-ray radiation under normal incidence conditions laserinduced periodic surface structures (LIPSS) are formed on $\mathrm{x}$-ray laser-illuminated surfaces only very scarcely $[44,45]$; this should be of use if a mask or interference pattern is transferred onto the surface because LIPSS-like modulation does not modify the pattern externally delivered on the sample surface.

Of course, the abovementioned applications achieve a practical relevance only if a compact FEL device is developed and implemented into a standard laboratory environment. Although such a device is not yet available, its development takes place within numerous projects attracting the attention of industry (see, for example, Refs. [46-50] and references therein). (c) Last but not least, the observed phenomena are relevant also for an estimation of possible damage to elements of x-ray optics exposed to many pulses generated at sources of the fourth generation. The single-shot damage thresholds can be often reliably predicted, easily measured, and well understood. Materials damage induced by accumulating a large number of short-wavelength laser pulses seems to be much more complicated and lesser understood phenomenon $[12,13,15,16]$. Although PMMA is a radiation-sensitive material, which is not used in $\mathrm{x}$-ray optics frequently, the detailed analysis of its multiplepulse-induced damage should be of use for future theoretical and experimental treatment of radiation-resistant materials $\left(a-\mathrm{C}, \mathrm{Si}, \mathrm{B}_{4} \mathrm{C}, \mathrm{SiC}, \mathrm{Si}_{3} \mathrm{~N}_{4}, \mathrm{Pt}, \mathrm{Au}, \mathrm{Be}\right)$ widely spread in the area of $\mathrm{x}$-ray optics manufacturing. We should also keep in mind that the irradiation experiments described in this paper represent a first step only because they are obtained at a relatively low repetition rate $(30 \mathrm{~Hz})$. A prospective PMMA exposure to $\mathrm{MHz}$ pulse trains sheds light on a relationship between thermal and nonthermal processes occurring in the irradiated sample. For successful development and utilization of prospective compact high-repetition-rate short-wavelength FEL sources, all the materials, manufacturing, and exposure issues must be carefully treated to make possible a survival of an optical element illuminated by multiple $>\mathrm{MHz}$ pulse trains.

Of course, there are more areas of a possible application of the above-reported results, e.g., durability of the first walls of prospective inertial fusion reactors [51], radiation damage to samples probed by FEL pulses [52,53], and high-dose-rate radiation biophysics [54], but their detailed discussion lies behind the scope of this paper.

\section{CONCLUSIONS}

Efficient material erosion is observed when an organic polymer is exposed to multiple $\mathrm{x}$-ray laser shots at a fluence level below the single-shot ablation threshold. Singlephoton radiolytical effects (especially polymer-chain scissions initiated by energetic photons, photoelectrons, and secondary electrons) are responsible for the subthreshold, desorptionlike damage in the material. Remarkably, the measured erosion rates (etch rates) are much greater than those typical for conventional long wavelength lasers and synchrotron radiation.

The high efficiency of the erosion is caused by the high local concentration of incoming photons resulting in a correlation of the single-photon interaction events and the following structure-breaking processes. The subthreshold damage exhibits nonlinear dose dependence of at least two types. The first one is represented by the nonlinear dependence of the depth of eroded area on the number of accumulated shots at the same level of fluence. Such an effect is assigned to the increase in the local density of 
$\mathrm{C}=\mathrm{C}$ double bonds in the irradiated area, which implies a higher radiation resistance of modified material.

The second type of nonlinearity is in the decrease in desorption rate with increasing fluences. This is found to be caused likely by different ratios between relative content of $1 \omega$ and $3 \omega$ component on the sample surface for different pressure settings of gas attenuator.

In general, these nonlinearities are caused by radiationdriven reactions resulting in an increase of polymer molecular weight, i.e., cross-linking, competing with the chain scissions and inhibition of efficient transport of lowmolecular fragments toward the surface and from the surface into the vacuum.

The dependence of the depths of eroded craters can be simulated with a model proposed for the interaction of PMMA with high-order harmonics (both the pulse and the photon energy lower than for FEL) even when the LCLS free-electron laser is used as the source of radiation. Another model based on a stochastic nature of the process is developed and tested on a prediction of the crater evolution. Both models fit the experiment very well in the case of an irradiation by a high number of pulses accumulated at a high fluence. However, the first model does not agree with the experimental results obtained for the first shots fired onto the surface at the lowest fluence while the new model fits them well.

\section{ACKNOWLEDGMENTS}

This work is financially supported by the Czech Science Foundation (Grants No. 19-03314S and No. 2008452S), by the Czech Ministry of Education, Youth and Sports (Grants No. LTT17015 and No. EF16_013/0001552 [OP VVV Project CZ.02.1.01/0.0/0.0/16-013/0001552ERDF]) and by the National Science Centre, Poland (Grant Agreement No. 2011/03/B/ST3/02453). We acknowledge a financial support from the European Union through the Future and Emerging Technologies (FET) Open H2020: PETACom (Grant No. 829153), the French Ministry of Research through the 2016 project "High rEpetition rate Laser for Lensless Imaging in the Xuv (HELLIX)" and the DGA RAPID grant "SWIM", the LABEX PALM (ANR10-LABX-0039-PALM) through the grants "Plasmon-X", HILAC and STAMPS and, finally, the Action de Soutien à la Technologie et à la Recherche en Essonne (ASTRE) program through the "NanoLight" grant are also acknowledged. This work is performed under the auspices of the U.S. Department of Energy by Lawrence Livermore National Laboratory under Contract DE-AC5207NA27344.

[1] Y. Zhang, Synchrotron radiation direct photo-etching of polymers, Adv. Polym. Sci. 168, 291 (2004).
[2] R. F. Haglund, Jr., Microscopic and mesoscopic aspects of laser-induced desorption and ablation, Appl. Surf. Sci. 96-98, 1 (1996).

[3] P. Jaeglé, Coherent Sources of XUV Radiation: Soft X-ray Lasers and High-Order Harmonic Generation (SpringerVerlag, Berlin-Heidelberg-New York, 2006).

[4] P. Schmüser, M. Dohlus, J. Rossbach, and C. Behrens, Free-electron Lasers in the Ultraviolet and X-ray Regime, 2nd Ed (Springer-Verlag, Heidelberg-New YorkDordrecht-London, 2014).

[5] K. J. Kim, Z. Huang, and R. Lindberg, Synchrotron Radiation and Free-Electron Lasers (Cambridge University Press, Cambridge, 2017).

[6] J. J. Rocca, Table-top soft x-ray lasers, Rev. Sci. Instrum. 70, 3799 (1999).

[7] H. Daido, Review of soft x-ray laser researches and developments, Rep. Prog. Phys. 65, 1513 (2002).

[8] G. J. Tallents, The physics of soft x-ray lasers pumped by electron collisions in laser plasmas, J. Phys. D 36, R259 (2003)

[9] P. Jaeglé and S. Suckewer, X-ray laser: Past, present, and future, Laser Phys. Lett. 6, 411 (2009).

[10] L. Juha, M. Bittner, D. Chvostova, J. Krasa, Z. Otcenasek, A. R. Präg, J. Ullschmied, Z. Pientka, J. Krzywinski, J. B. Pelka, et al., Ablation of organic polymers by 46.9-nm laser radiation, Appl. Phys. Lett. 86, 034109 (2005).

[11] J. Chalupský, L. Juha, V. Hájková, J. Cihelka, L. Vyšín, J. Gautier, J. Hajdu, S. P. Hau-Riege, M. Jurek, J. Krzywinski, et al., Non-thermal desorption/ablation of molecular solids induced by ultra-short soft x-ray pulses, Opt. Express 17, 208 (2009).

[12] I. A. Makhotkin, R. Sobierajski, J. Chalupský, K. Tiedtke, G. de Vries, M. Störmer, F. Scholze, F. Siewert, R. W. E. van de Kruijs, I. Milov, et al., Experimental study of EUV mirror radiation damage resistance under long-term free-electron laser exposures below the single-shot damage threshold, J. Synchrotron. Rad. 25, 77 (2018).

[13] R. Sobierajski, I. Jacyna, P. Dłużewski, M. T. Klepka, D. Klinger, J. B. Pełka, T. Burian, V. Hájková, L. Juha, K. Saksl, et al., Role of heat accumulation in the multi-shot damage of silicon irradiated with femtosecond XUV pulses at a $1 \mathrm{MHz}$ repetition rate, Opt. Express 24, 15468 (2016).

[14] T. Koyama, H. Yumoto, T. Miura, K. Tono, T. Togashi, Y. Inubushi, T. Katayama, J. Kim, S. Matsuyama, M. Yabashi, et al., Damage threshold of coating materials on X-ray mirror for x-ray free electron laser, Rev. Sci. Instrum. 87, 051801 (2016).

[15] J. Krzywinski, D. Cocco, S. Moeller, and D. Ratner, Damage threshold of platinum coating used for optics for selfseeding of soft x-ray free electron laser, Opt. Express 23, 5397 (2015).

[16] L. Juha, V. Hájková, J. Chalupský, V. Vorlíček, A. Ritucci, A. Reale, P. Zuppella, and M. Stormer, Radiation damage to amorphous carbon thin films irradiated by multiple 46.9 nm laser shots below the single-shot damage threshold, J. Appl. Phys. 105, 093117 (2009).

[17] J. Chalupský, P. Boháček, T. Burian, V. Hájková, S. P. Hau-Riege, P. A. Heimann, L. Juha, M. Messerschmidt, S. P. Moeller, B. Nagler, et al., Imprinting a Focused X-Ray Laser Beam to Measure its Full Spatial Characteristics, Phys. Rev. Appl. 4, 014004 (2015). 
[18] H. Winick, K. Bane, R. Boyce, J. Cobb, G. Loew, P. Morton, H.-D. Ntihn, J. Paterson, P. Pianetta, T. Raubenheimer, et al., Short wavelength FELs using the SLAC linac, Nucl. Instrum. Meth. Phys. Res. A 347, 199 (1994).

[19] E. L. Saldin, E. A. Schneidmiller, and M. V. Yurkov, The Physics of Free Electron Lasers (Springer-Verlag, BerlinHeidelberg-New York, 2000).

[20] P. Emma, R. Akre, J. Arthur, R. Bionta, C. Bostedt, J. Bozek, A. Brachmann, P. Bucksbaum, R. Coffee, F.-J. Decker, et al., First lasing and operation of an ångstromwavelength free-electron laser, Nat. Photonics 4, 641 (2010).

[21] B. L. Henke, E. M. Gullikson, and J. C. Davis, X-ray interactions: Photoabsorption, scattering, transmission, and reflection at $\mathrm{E}=50-30,000 \mathrm{eV}, \mathrm{Z}=1-92$, At. Data Nucl. Data Tables 54, 181 (1993).

[22] A. Barty, R. Soufli, T. McCarville, S. L. Baker, M. J. Pivovaroff, P. Stefan, and R. M. Bionta, Predicting the coherent $\mathrm{X}$-ray wavefront focal properties at the linac coherent light source (LCLS) X-ray free electron laser, Opt. Express 17, 15508 (2009).

[23] J. D. Bozek, AMO instrumentation for the LCLS x-ray FEL, Eur. Phys. J., Spec. Top. 169, 129 (2009).

[24] M. de Grazia: Applications du rayonnement harmonique à l'interaction UVX-solide, dissertation, L'Université Paris XI (Orsay, France), 2007, 216pp.

[25] M. De Grazia, H. Merdji, T. Auguste, B. Carré, J. Gaudin, G. Geoffroy, S. Guizard, F. Krejci, J. Kuba, J. Chalupský, et al., Desorption mechanisms in PMMA irradiated by high order harmonics, Proc. SPIE 8077, 80770L-1 (2011).

[26] J. Chalupský, J. Krzywinski, L. Juha, V. Hájková, J. Cihelka, T. Burian, L. Vyšín, J. Gaudin, A. Gleeson, M. Jurek, et al., Spot size characterization of focused nonGaussian x-ray laser beams, Opt. Express 18, 27836 (2010).

[27] J. Chalupský, T. Burian, V. Hájková, L. Juha, T. Polcar, J. Gaudin, M. Nagasono, R. Sobierajski, M. Yabashi, and J. Krzywinski. Fluence scan: An unexplored property of a laser beam Opt. Express 21, 26363 (2013).

[28] E. M. Lehockey, I. Reid, and I. Hill, I. Physical and chemical aspects of PMMA vapour development, Nucl. Instrum. Meth. Phys. Res. B 46, 364 (1990).

[29] E. M. Lehockey, I. Reid, and I. Hill, The radiation chemistry of poly(methyl methacrylate) polymer resists, J. Vac. Sci. Technol., A 6, 828 (1997).

[30] X. Zhang, C. Jacobsen, S. Lindaas, and S. Williams, Exposure strategies for polymethyl methacrylate from in situ x-ray absorption near edge structure spectroscopy, J. Vac. Sci. Technol., B 13, 1477 (1995).

[31] A. F. G. Leontowich, A. P. Hitchcock, T. Tyliszczak, M. Weigand, J. Wang, and C. Karunakaran, Accurate dosimetry in scanning transmission X-ray microscopes via the cross-linking threshold dose of poly(methyl methacrylate), J. Synchrotron. Rad. 19, 976 (2012).

[32] M. Ross and A. Charlesby, The effect of pile radiation on poly(methyl methacrylate), Atomics 4, 189 (1953).

[33] P. Alexander, M. Ross, and A. Charlesby, The degradation of solid polymethylmethacrylate by ionizing radiation, Proc. Roy. Soc. (London) A 223, 392 (1954).

[34] A. Charlesby, Atomic Radiation and Polymers (Pergamon Press, Oxford-London-NY-Paris, 1960), Chap. 19.
[35] H. Hiraoka, Radiation chemistry of poly(methacrylates), IBM J. Res. Dev. 21, 121 (1977).

[36] H. Srinivasan, B. Brarer, and K. G. Kasey, Nature of "incubation pulses" in the ultraviolet laser ablation of polymethyl methacrylate, J. Appl. Phys. 68, 1842 (1990).

[37] D. Ashkenasi, M. Lorenz, R. Stoian, and A. Rosenfeld, Surface damage threshold and structuring of dielectrics using femtosecond laser pulses: The role of incubation, Appl. Surf. Sci. 150, 101 (1999).

[38] A. Bartnik, H. Fiedorowicz, R. Jarocki, L. Juha, J. Kostecki, R. Rakowski, and M. Szczurek, Strong temperature effect on X-ray photo-etching of polytetrafluoroethylene using a $10 \mathrm{~Hz}$ laser-plasma radiation source based on a gas puff target, Appl. Phys. B 82, 529 (2006).

[39] R. Kirchner and H. Schmidt, Thermal reflow of polymers for innovative and smart 3D structures: A review, Mater. Sci. Semicond. Proc. 92, 58 (2019).

[40] M. Toufarová, V. Hájková, J. Chalupský, T. Burian, J. Vacík, V. Vorlíček, L. Vyšín, J. Gaudin, N. Medvedev, B. Ziaja, et al., Contrasting behavior of covalent and molecular carbon allotropes exposed to extreme ultraviolet and soft $\mathrm{x}$-ray free-electron laser radiation, Phys. Rev. B 96, 214101 (2017).

[41] G. Vaschenko, A. Garcia Etxarri, C. S. Menoni, J. J. Rocca, O. Hemberg, S. Bloom, W. Chao, E. H. Anderson, D. T. Attwood, Y. Lu, et al., Nanometer-scale ablation with a table-top soft x-ray laser, Opt. Lett. 31, 3615 (2006).

[42] T. Mocek, B. Rus, M. Kozlová, J. Polan, P. Homer, L. Juha, V. Hájková, and J. Chalupský, Single-shot soft x-ray laser-induced ablative microstructuring of organic polymer with demagnifying projection, Opt. Lett. 33, 1087 (2008).

[43] K. Kolacek, J. Schmidt, J. Straus, O. Frolov, L. Juha, and J. Chalupsky, Interaction of extreme ultraviolet laser radiation with solid surface: Ablation, desorption, nanostructuring, Proc. SPIE 9255, 92553U (2014)

[44] B. Steeg, L. Juha, J. Feldhaus, S. Jacobi, R. Sobierajski, C. Michaelsen, A. Andrejczuk, and J. Krzywinski, Total reflection amorphous carbon mirrors for vacuum ultraviolet free electron lasers, Appl. Phys. Lett. 84, 657 (2004).

[45] L. Juha, M. Bittner, D. Chvostová, J. Krása, M. Kozlová, M. Pfeifer, J. Polan, A. R. Präg, B. Rus, M. Stupka, et al., Short-wavelength ablation of molecular solids: Pulse duration and wavelength effects, J. Microlith. Microfab. Microsyst. 4, 033007 (2005).

[46] C. Pagani, E. L. Saldin, E. A. Schneidmiller, and M. V. Yurkov, Design considerations of $10 \mathrm{~kW}$-scale extreme ultraviolet SASE FEL for lithography, Nucl. Instrum. Meth. Phys. Res. A 463, 9 (2001).

[47] G. Dattoli, P. L. Ottaviani, A. Renieri, S. G. Biedron, H. P. Freund, and S. V. Milton, A compact free electron laser device operating in the UV-soft x-ray region, Opt. Commun. 232, 319 (2004).

[48] Y. Socol, G. N. Kulipanov, A. N. Matveenko, O. A. Shevchenko, and N. A. Vinokurov, Compact 13.5-nm freeelectron laser for extreme ultraviolet lithography, Phys. Rev. Spec. Top. - Accel. Beams 14, 040702 (2011).

[49] E. R. Hosler, O. Wood, and W. Barletta, Free-electron laser emission architecture impact on extreme ultraviolet 
lithography, J. Micro-Nanolithogr. MEMS and MOEMS 16, 041009 (2017).

[50] M. E. Couprie, Towards compact free electron-laser based on laser plasma accelerators, Nucl. Instrum. Meth. Phys. Res. A 909, 5 (2018).

[51] J. Straus, K. Kolacek, J. Schmidt, O. Frolov, M. Vilemova, J. Matejicek, A. Jager, L. Juha, M. Toufarová, A. Choukourov, et al., Response of fusion plasma-facing materials to nanosecond pulses of extreme ultraviolet radiation, Laser Part. Beams 36, 293 (2018).
[52] K. Nass, Radiation damage in protein crystallography at x-ray free-electron lasers, Acta Cryst. D 75, 211 (2019).

[53] N. H. Chapman, X-ray free-electron lasers for the structure and dynamics of macromolecules, Annu. Rev. Biochem 88, 35 (2019).

[54] L. Vyšín, T. Burian, E. Ukraintsev, M. Davídková, M. E. Grisham, S. Heinbuch, J. J. Rocca, and L. Juha, Dose-rate effects in breaking DNA strands by short pulses of extreme ultraviolet radiation, Rad. Res. 189, 466 (2018). 